\title{
An Immunosuppressive Status Classifier to Predict Recurrence and Assist in Decision Regarding Postoperative Adjuvant Treatment in Gastric Cancer
}

Jia-Bin Wang

Fujian Medical University Union Hospital

Qing-Zhu Qiu

Fujian Medical University Union Hospital

Ning-Zi Lian

Fujian Medical University Union Hospital

Hua-Gen Wang

Fujian Medical University Union Hospital

Qiao-Ling Zheng

Fujian Medical University Union Hospital

\section{Ying-Hong Yang}

Fujian Medical University Union Hospital

\section{Yu-Bin Ma}

Qinghai University Medical College

Ya-Jun Zhao

The First Affiliated Hospital of USTC: Anhui Provincial Hospital

\section{Ping Li}

Fujian Medical University Union Hospital

Jian-Xian Lin

Fujian Medical University Union Hospital

Jun Lu

Fujian Medical University Union Hospital

Qi-Yue Chen

Fujian Medical University Union Hospital

\section{Long-Long Cao}

Fujian Medical University Union Hospital

Mi Lin

Fujian Medical University Union Hospital

\section{Chang-Ming Huang}

Fujian Medical University Union Hospital

Jian-Wei Xie ( $\nabla$ xjwhw2019@163.com ) 


\section{Research}

Keywords: Recurrence, Immunosuppressive indicators, Immune contexture, Adjuvant therapy, Gastric cancer.

Posted Date: July 19th, 2021

DOI: https://doi.org/10.21203/rs.3.rs-684190/v1

License: (c) (1) This work is licensed under a Creative Commons Attribution 4.0 International License. Read Full License 


\section{Abstract}

Background: Suppression of the immune microenvironment is a crucial cause of postoperative tumor recurrence. We constructed an immune infiltration classifier based on immunosuppressive indicators to predict recurrence and guide postoperative treatment for gastric cancer (GC).

Methods: Immunohistochemical analysis was performed for $825 \mathrm{GC}$ tissues to evaluate immunosuppressive indicators. An immunosuppressive recurrence score (IRS) based on six immunosuppressive indicators was determined using the Lasso Cox method to predict recurrence outcomes. The association between immune infiltration and IRS was assessed using immunohistochemistry and multiplexed immunofluorescence staining. A nomogram predicting recurrence-free survival (RFS) was constructed by integrating IRS and significant clinicopathological features using the Cox regression model.

Results: The IRS and IRS-based nomogram showed remarkable accuracy and reliability for predicting the recurrence outcome. Moreover, elevated IRS was associated with locoregional recurrence and failure of postoperative adjuvant chemotherapy. We also identified that the increased IRS indicated the inhibition of anti-tumor effect of $\mathrm{CD}^{+}$tumor-infiltrating lymphocytes (TILs) in the invasive margin (IM).

Conclusion: IRS can predict the recurrence outcome of GC patients by comprehensively distinguishing the immune infiltration status, which assists in the selection of different adjuvant treatment options

\section{Background}

Recurrence is the main reason for the failure of postoperative treatment for gastric cancer (GC) [1]. It includes three major modes: local recurrence, peritoneal metastasis, and distant recurrence $[2,3]$. Approximately $40-60 \%$ of GC patients have a poor prognosis owing to recurrence $[4,5]$. Adjuvant chemotherapy remains an important treatment for reducing the risk of recurrence in patients with GC [6, 7], and it can kill tumor cells directly and increase their susceptibility to immune effects, thereby exerting tumoricidal activity [8-10]. Nevertheless, resistance to chemotherapy limits its clinical application, and some patients still relapse after chemotherapy. Immunotherapy has shown remarkable antitumor effects in the treatment of various solid tumors, and for some tumors, it has become a first-line treatment [1116]. Anti-PD-1 immunotherapy in patients with GC can significantly improve survival $[17,18]$. However, it is worth noting that immunotherapy still lacks a high response rate in GC [19], and this may be attributed to the heterogeneity of the immune microenvironment $[20,21]$. Tumors with increased immune cell infiltration can benefit from immunotherapy [20, 22]. Therefore, classifying different immune states may be key in determining treatment options and predicting prognosis in GC.

Suppression of the immune microenvironment is a considerable factor leading to recurrence, which may be because the tumor escapes from destruction by the immune system [23-26]. This suppression of the immune microenvironment is inseparable from the interaction of many factors such as tumor-infiltrating lymphocytes (TILs). CD8 ${ }^{+}$TILs are the effectors that eliminate tumors, and their suppression is often 
linked to poor outcomes such as local recurrence [27]. The high expression of immune checkpoints on CD8 ${ }^{+}$TILs, such as PD-1 and TIM-3, is a sign of T cell exhaustion and the co-expression of PD- 1 and TIM3 is related to hypo-functional TILs [28-30]. The increased infiltration of CD8 ${ }^{+}$TILs in the center of the tumor (CT) or invasive margin (IM) is related to improved diseases-free survival (DFS) [31-33].

Tumor cell-intrinsic factors such as immunosuppressive indicators might also be initiators of this immunosuppressive microenvironment [34]. These immunosuppressive indicators inhibit the activation and downregulation of $\mathrm{T}$ cell infiltration by reprogramming immune cells and inducing them to release inhibitory factors [35-37]. They can also reduce the sensitivity to cytotoxic T lymphocytes to avoid immune destruction or bind to inhibitory receptors on the surface of lymphocytes to mediate lymphocyte apoptosis [38, 39]. However, the expression of immunosuppressive indicators is heterogeneous. Different types of cancers or different patients with the same type of cancer express different immunosuppressive indicators. It is unclear which immunosuppressive indicators are expressed in GC and whether they are valuable for predicting the prognosis of GC patients. This limits the applicability of immunosuppressive indicators as prognostic markers. The expression of PD-L1, as a basis for predicting treatment benefits, is not stable, and only few PD-L1-negative patients receive some relief from treatment [40]. This shows that immunosuppressive indicators can be used for predicting the prognosis of GC patients and evaluating adjuvant treatment options, but the accuracy based on a single molecule is not satisfactory. Our previous research revealed that the signature based on multiple immunosuppressive indicators showed a great prognostic value in predicting the prognosis of GC patients and distinguishing GC patients who can benefit from chemotherapy [41]. Based on our previous studies, we further explored the potential of the signature based on immunosuppressive indicators in predicting the recurrence status and recurrence patterns and guiding immunotherapy.

In the present study, we integrated immunosuppressive indicators with prognostic value in GC patients to construct a signature immunosuppressive recurrence score (IRS) to classify patients with GC with different recurrence risks. We then used this as a basis to predict the recurrence patterns that patients with different recurrence risks have. We further verified the ability of the IRS to distinguish different immune characteristics to determine the potential benefits of adjuvant chemotherapy and immunotherapy. Up to a point, IRS can guide surgeons in selecting adjuvant treatment options for GC patients according to different recurrence risks, including immunotherapy and chemotherapy. At the same time, the IRS can help the development of follow-up strategies and inspection plans to enable patients to obtain better outcomes.

\section{Methods}

\section{Patients and tissue specimens}

This study included 825 GC patient tissues collected between January 2010 and October 2015. Among them, 627 patient tissues from Fujian Medical University Union Hospital were divided into the training cohort $(n=418,2010-2013)$ and internal validation cohort $(n=209,2013-2015)$ according to the time of 
operation. 198 patients from Qinghai University Hospital (110 patients) and the First Affiliated Hospital of University of Science and Technology of China (88 patients) were included in the external validation cohort. Detailed inclusion and exclusion criteria are described in the Supplementary Methods.

\section{Definition and classification of recurrence}

Recurrence was defined as the presence of a tumor showing GC cells confirmed on a tissue biopsy or the presence of imaging features that are highly indicative of tumor recurrence. Recurrence was categorized as locoregional recurrence, peritoneal implantation, or distant metastasis. Locoregional recurrence included recurrence in the gastric bed, anastomoses, and perigastric lymph nodes, and recurrence in the duodenum around the stomach was defined as local recurrence, belonging to the "other" category.

\section{Immunohistochemistry}

Seven immunosuppressive indicators based on previous research for immunohistochemistry analysis: SIGLEC6, CD44, CEACAM1, CD155, HMGB1, NECTIN2 and ADENOSINE [41]. Scoring criteria for immunosuppressive indicators as follows. In five randomly selected fields, the average percentage and intensity of positive cells were evaluated to determine protein expression levels. The scoring criteria (Supplementary Figure S2) were as follows: the staining intensity was categorized as 0 (no staining), 1 (weak staining, light yellow), 2 (medium staining, yellow to brown), or 3 (strong staining, brown), and the proportion of positive tumor cells was categorized as 0 ( $\leq 5 \%$ positive cells), $1(6-25 \%), 2(26-50 \%)$, or 3 $(\geq 51 \%)$. The staining intensity score was multiplied by the proportional staining score (total score: $0-9$ ) to calculate the final score of expression. Patients with a final score $<4$ were included in the lowexpression group and those with a score $\geq 4$ in the high-expression group.

To assess the immune contexture of GC patients, we analyzed total immune cells (CD45), T lymphocytes (CD3), cytotoxic T lymphocytes (CD8) and activated and memory T lymphocytes. To evaluate the infiltration of immune cells, five representative and independent fields at $\times 200$ magnification were captured at the CT and IM of each tissue. An example of this procedure is shown in Supplementary Figure S13. Next, we used the "measurement" plug-in of Image Pro Plus software (ver. 6.0; Inc., USA) to assist in marker counting to obtain the number of positive cells in the field. The average number of positive cells in the five fields was divided by the area of the field to obtain the infiltration density of immune cells in the CT and IM.

To determine the microsatellite instability (MSI) and Epstein-Barr virus (EBV) status of the patients, we performed MLH1, MSH2, MSH6, PMS2 immunohistochemical staining, and EBER (ISH-6021; ZSGB-BIO) in situ hybridization. The evaluation criteria of MSI and EBV status are shown in Supplementary Fig. 7 and Supplementary Methods.

Two experienced pathologists, blinded to the clinicopathological characteristics and prognosis of the patients, independently scored all samples. When scoring immune checkpoints and evaluating the MSI and EBV status, approximately $93 \%$ of the scores were completely concordant. When the scores of the two independent pathologists diverged, another pathologist reviewed the results and chose one of the 
scores of the first two pathologists, or the three pathologists arrived at consensus. Details of the antibodies used in this study are provided in Supplementary Table S7.

\section{Building the IRS using the lasso Cox regression model}

The lasso Cox regression model was used to integrate seven meaningful indicators and eliminate the offset caused by the correlation between variables to build a signature for predicting recurrence in the training cohort ( $n=418)$. We use the "glmnet" package of R software (ver. 4.0.0) to analyze the data using the lasso Cox regression model. The three risk groups were distinguished according to the optimal cutoff value of the IRS using X-tile software (ver. 3.6.1).

\section{Multiplexed immunofluorescence staining and analysis}

To determine differences in the tumor microenvironment in different IRS risk groups, we performed multiplexed immunofluorescence staining to identify the expression of CD8, PD-1, and TIM-3 using the Opal kit (Perkin-Elmer, USA) in $45 \mathrm{GC}$ tissues. In the three risk subgroups of the internal validation cohort, each GC patients was assigned a random number in each subgroups, and the top 15 patients (ranked from small to large) in each group were selected by random number, for a total of 45 cases.

We used the Mantra System (Perkin-Elmer, USA) to scan the multiplexed immunofluorescence stained tissues at $\times 200$ magnification and InForm image analysis software (Perkin-Elmer, USA) to quantify positive cells automatically. To ensure the accuracy of the results, two pathologists verified the results individually using the abovementioned method. When the difference between the results of the software and those of the two pathologists was within $5 \%$, the average of the two results was obtained. If the difference exceeded $5 \%$, the two pathologists reassessed the results and asked the third pathologist to evaluate the results. Finally, the third pathologist decided which result would be accepted. The detailed steps are shown in the Supplementary Methods.

\section{Statistical analysis}

SPSS ver. 26.0 (IBM Inc., USA), R software (ver. 4.0.0) were used to process all data. We used the MannWhitney $U$ test to compare two groups and the Kruskal-Wallis test to perform multiple comparisons. The categorical variables of clinicopathological characteristics were compared using the $\chi^{2}$ test or Fisher's exact test. Nonparametric correlation analyses were performed using Spearman's tests, respectively. Kaplan-Meier survival analysis with the log-rank test was used to estimate recurrence-free survival (RFS). Cox proportional hazard model was used to confirm the association between relevant clinicopathological variables and RFS. Significant variables after univariate Cox analysis were included in the multivariate Cox analysis. Statistical significance was set at $P<0.05$.

\section{Results}

\section{Patient characteristics and construction of the IRS}


The clinicopathological data of $825 \mathrm{GC}$ patients are presented in Table 1 and the flow chart of this study is shown in Supplementary Fig. 1. 
Table 1

Clinicopathological Characteristics of the GC Patients in Each Cohorts.

\begin{tabular}{|c|c|c|c|c|c|c|}
\hline \multirow[t]{3}{*}{ Variable } & \multicolumn{4}{|c|}{ Internal Cohort } & \multirow{2}{*}{\multicolumn{2}{|c|}{$\begin{array}{l}\text { External } \\
\text { Validation Cohort }\end{array}$}} \\
\hline & \multicolumn{2}{|c|}{ Training Cohort } & \multicolumn{2}{|c|}{ Validation Cohort } & & \\
\hline & $\mathbf{N}$ & $\%$ & $\mathbf{N}$ & $\%$ & $\mathbf{N}$ & $\%$ \\
\hline Total Patients & 418 & - & 209 & - & 198 & - \\
\hline \multicolumn{7}{|l|}{ Age(years) } \\
\hline$\leq 65$ & 246 & 58.9 & 120 & 57.4 & 140 & 70.7 \\
\hline$>65$ & 172 & 41.1 & 89 & 42.6 & 58 & 29.3 \\
\hline \multicolumn{7}{|l|}{ Sex } \\
\hline Female & 98 & 23.4 & 57 & 27.3 & 60 & 30.3 \\
\hline Male & 320 & 76.7 & 152 & 72.7 & 138 & 69.7 \\
\hline \multicolumn{7}{|l|}{ BMI } \\
\hline$\leq 25$ & 351 & 84.0 & 178 & 85.2 & - & - \\
\hline$>25$ & 67 & 16.0 & 31 & 14.8 & - & - \\
\hline \multicolumn{7}{|l|}{ Resection type } \\
\hline Part gastrectomy & 183 & 43.8 & 97 & 46.4 & 144 & 72.7 \\
\hline Total gastrectomy & 235 & 56.2 & 112 & 53.6 & 54 & 27.3 \\
\hline \multicolumn{7}{|l|}{ Tumor size } \\
\hline$\leq 50 \mathrm{~mm}$ & 230 & 55.0 & 112 & 53.6 & 137 & 69.2 \\
\hline$>50 \mathrm{~mm}$ & 188 & 45.0 & 97 & 46.4 & 61 & 30.8 \\
\hline \multicolumn{7}{|l|}{ Tumor Location } \\
\hline Cardia & 101 & 24.2 & 52 & 24.9 & - & - \\
\hline Body & 88 & 21.1 & 32 & 15.3 & - & - \\
\hline Antrum & 186 & 44.6 & 93 & 44.5 & - & - \\
\hline Whole & 43 & 10.1 & 32 & 15.3 & - & - \\
\hline \multicolumn{7}{|l|}{ Grade } \\
\hline Low & 189 & 45.2 & 105 & 50.2 & 67 & 33.8 \\
\hline Middle + High & 144 & 34.5 & 68 & 32.6 & 76 & 38.4 \\
\hline Mix & 67 & 16.0 & 33 & 15.8 & 52 & 26.3 \\
\hline
\end{tabular}




\begin{tabular}{|c|c|c|c|c|c|c|}
\hline \multirow[t]{3}{*}{ Variable } & \multicolumn{4}{|c|}{ Internal Cohort } & \multirow{2}{*}{\multicolumn{2}{|c|}{$\begin{array}{l}\text { External } \\
\text { Validation Cohort }\end{array}$}} \\
\hline & \multicolumn{2}{|c|}{ Training Cohort } & \multicolumn{2}{|c|}{ Validation Cohort } & & \\
\hline & $\mathbf{N}$ & $\%$ & $\mathbf{N}$ & $\%$ & $\mathbf{N}$ & $\%$ \\
\hline Unknow & 18 & 4.3 & 3 & 1.4 & 3 & 1.5 \\
\hline \multicolumn{7}{|c|}{ Depth of invasion } \\
\hline T1 & 37 & 8.8 & 36 & 17.2 & 36 & 18.2 \\
\hline $\mathrm{T} 2$ & 47 & 11.2 & 22 & 10.5 & 41 & 20.7 \\
\hline T3 & 151 & 36.1 & 92 & 44.0 & 17 & 8.6 \\
\hline T4 & 183 & 43.8 & 59 & 28.2 & 104 & 52.5 \\
\hline \multicolumn{7}{|c|}{ Lymph node metastasis } \\
\hline No & 94 & 22.5 & 71 & 34.0 & 94 & 47.5 \\
\hline N1 & 76 & 18.2 & 40 & 19.1 & 34 & 17.1 \\
\hline N2 & 88 & 21.0 & 32 & 15.3 & 31 & 15.7 \\
\hline N3 & 160 & 38.3 & 66 & 31.6 & 39 & 19.7 \\
\hline \multicolumn{7}{|l|}{ AJCC(7th) } \\
\hline I & 55 & 13.2 & 44 & 21.1 & 57 & 28.8 \\
\hline ॥ & 113 & 27.0 & 64 & 30.6 & 53 & 26.8 \\
\hline III & 250 & 59.8 & 101 & 48.3 & 88 & 44.4 \\
\hline \multicolumn{7}{|l|}{ MSI status } \\
\hline MSS/MSI-L & 337 & 80.6 & 169 & 80.9 & 144 & 72.7 \\
\hline MSI-H & 81 & 19.4 & 40 & 19.1 & 54 & 27.3 \\
\hline \multicolumn{7}{|l|}{ EBV status } \\
\hline Negative & 393 & 94.0 & 196 & 93.8 & 189 & 95.5 \\
\hline Positive & 25 & 6.0 & 13 & 6.2 & 9 & 4.5 \\
\hline \multicolumn{7}{|c|}{ Adjuvant Chemotherapy } \\
\hline Yes & 221 & 52.9 & 110 & 52.6 & 100 & 50.5 \\
\hline No & 197 & 47.1 & 99 & 47.4 & 98 & 49.5 \\
\hline \multicolumn{7}{|l|}{ CEA } \\
\hline Normal & 346 & 82.8 & 191 & 91.4 & - & - \\
\hline
\end{tabular}




\begin{tabular}{|lllllll|}
\hline Variable & \multicolumn{3}{l}{ Internal Cohort } & \multicolumn{3}{c|}{ External } \\
\cline { 2 - 7 } & \multicolumn{2}{l}{ Training Cohort } & \multicolumn{2}{c|}{ Validation Cohort } & \multicolumn{2}{c|}{ Validation Cohort } \\
\cline { 2 - 7 } & $\mathbf{N}$ & $\%$ & $\mathbf{N}$ & $\%$ & $\mathbf{N}$ & $\%$ \\
\hline Elevated & 72 & 17.2 & 18 & 8.6 & - & - \\
\hline CA19-9 & & & & & & \\
\hline Normal & 330 & 78.9 & 166 & 79.4 & - & - \\
\hline Elevated & 88 & 21.1 & 43 & 20.6 & - & - \\
\hline
\end{tabular}

To verify the prognostic value of immunosuppressive indicators (SIGLEC6, CD44, CEACAM1, CD155, HMGB1, NECTIN2, and ADENOSINE) for RFS, we used immunohistochemical methods to evaluate the expression of the seven indicators in GC specimens from 827 patients from our center (Fig. 1a). As we hypothesized, the Kaplan-Meier curves of the seven indicators for RFS all showed that the 5-year recurrence rate of the high-expression was lower than that of the low-expression (S3-5 Fig). As reported in our previous research[41], there was a correlation between the seven immunosuppressive indicators. To reduce the impact of correlations between these seven indicators and construct a scoring system, we used the LASSO Cox regression model to fit the RFS data from the training cohort. In the optimization model, six of the seven indicators were selected, and the selected six indicators and their corresponding regression coefficients (Fig. 1b and c) were used to derive the model formula. The following formula was used for calculating the IRS for each patient: IRS $=0.17543 \times$ CEACAM1 + $0.04265 \times$ SIGLEC6 $+0.20185 \times$ CD44 + $0.05687 \times$ CD155 + $0.02017 \times$ ADNEOSINE $-0.0507 \times$ NECTIN2. In the formula generated by Lasso, the HMGB1 indicator was excluded, which was attributed to the correlation coefficient of 0 (less critical in the formula). To explore the clinical characteristics of GC patients with different IRS scores, we divided these patients into three subgroups (low-risk, 0-1.92; middle-risk, 1.93-3.02; and high-risk, 3.03-5) according to the cutoff point calculated using X-tile software (S6a-c Fig).

As mentioned above, we classified GC patients into three subgroups according to the IRS we constructed based on six immunosuppressive signatures. This provided the basis for our analysis of the clinical outcomes and infiltration of immune cells.

\section{Value of IRS in predicting recurrence}

Receiver operator characteristic (ROC) curves analysis show that the IRS could accurately predict the outcome of recurrence in different years (Fig. 2a to c). For the five-year recurrence rate, the three risk subgroups shown significant differences (Fig. $2 d$ to f). Similar results were obtained in patients with different clinicopathological factors (except for Stage I) in Supplementary Fig. S8 to S10. The univariate and multivariate analyses using Cox regression model showed that IRS was a powerful and independent factor for RFS (Supplementary Tables S3-S5). The $\chi^{2}$ test was performed in three cohorts to compare the differences in clinicopathological variables in different risk groups, and there was no significant difference except for TNM stage and CA19-9 level (Supplementary Tables S1 and S2). 
In conclusion, our research revealed that IRS is a powerful and independent factor and could significantly distinguish postoperative GC patients with different risks of recurrence. The value of IRS in predicting recurrence might not be affected by different TNM stages or the MSI and EBV status, indicating that IRS is widely applicable in GC.

\section{IRS was correlated with postoperative recurrence time}

We analyzed the composition of patients with different clinical outcomes among the three groups. In the high-risk, $45.2 \%$ of the total group had recurrence within 1 year, while in the low-risk, the percentage was only $2.7 \%$ and that in the middle-risk was between the other two groups. The results were also applicable for patients with recurrence within 2 and 3 years, but the opposite was observed in patients without recurrence $(P<0.001$, Fig. $2 \mathrm{~g}$ to i). The significant difference in recurrence status among the three risk groups led us to hypothesize that the IRS score is related to the patients' RFS. As expected, patients with early recurrence had higher IRS scores than those with late recurrence (Fig. 2j). We obtained similar results in the internal and external cohorts (Fig. 2k and I), and the lack of patients with recurrence after 5 years in the external cohort did not affect our conclusion. In summary, GC patients with higher IRS scores were more likely to relapse after surgery, and these patients relapsed within a shorter time.

\section{IRS predicts recurrence patterns}

The recurrence patterns in the training and internal validation cohorts are shown in Fig. 3a. To explore whether IRS can predict the patterns of recurrence, we compared the IRS scores of patients with and without locoregional recurrence among all patients who experienced recurrence. Figure $3 \mathrm{~b}$ and $\mathrm{c}$ show that patients with local recurrence had a significantly higher IRS $(P=0.002)$. However, patients with peritoneal and distant metastases did not show any difference (S11a and b Fig). We also found that among patients with locoregional recurrence (S11c Fig) shows different recurrence sites in patients with locoregional recurrence), patients with recurrence of perigastric lymph nodes had higher IRS scores than patients without recurrence of lymph nodes $(P=0.034$, Fig. 3d).

As such, the IRS may be able to predict different recurrence patterns. For patients with a higher IRS, postoperative local recurrence is more likely to occur, especially in the perigastric lymph node area. However, other areas should not be ignored, because in our analysis, patients in the locoregional group (red circle in Fig. 3a and the lymph nodes group (red circle in S11c Fig) also had other recurrence patterns or sites.

\section{IRS predicts the benefit of chemotherapy}

As shown in Supplementary Figure. S12, chemotherapy significantly reduced the recurrence rate in the middle-risk, while in the high-risk, patients failed to benefit from chemotherapy. In the low-risk, the overall recurrence rate was low, and chemotherapy use did not improve the recurrence rate. The results were consistent between the training cohort and the internal and external validation cohorts.

In our study, GC patients in the middle-risk were treated with chemotherapy to reduce the recurrence rate, and patients in the low-risk had a lower recurrence rate regardless of receiving chemotherapy. However, 
patients in the high-risk who received chemotherapy failed to achieve a better prognosis.

\section{IRS is linked to the infiltration of T lymphocytes in the IM}

To analyze the divergence in the infiltration status of immune cells between patients with different IRS, the density of several lymphocytes was measured. There was a large difference in the infiltration of immune cells between the CT and IM, therefore, these two areas were measured separately (Fig. 4a).

First, we evaluated the infiltration of leukocyte $\left(C D 45^{+}\right)$. However, in patients with different recurrence outcomes, there was no significant discrepancy in the infiltration of leukocyte, both in the IM or CT (S14S16 Fig).

Second, we narrowed down the scope of our research to TILs. In non-relapsed patients, a higher level of infiltration of $\mathrm{CD}^{+}$TILs was found (Fig. 4b). The density of $\mathrm{CD}^{+}{ }_{(\mathrm{IM})}$ TILs in the low-risk significantly exceeded that in the middle- and high-risk, but the middle-risk and the high-risk showed an equal level (Fig. 4c). The density of $\mathrm{CD}^{+}{ }_{(\mathrm{IM})}$ TILs showed a negative correlation with IRS in the low-risk and high-risk ( $P=0.003, P=0.001$, respectively), but this trend was the opposite in the high-risk $(P=0.012$, Fig. $4 \mathrm{~d})$.

The same analysis was performed for memory $\mathrm{T}$ lymphocytes $\left(\mathrm{CD} 45 \mathrm{RO}^{+}\right)$and cytotoxic $\mathrm{T}$ lymphocytes $\left(\mathrm{CD} 8^{+}\right)$. For patients with recurrence, the density of $\mathrm{CD} 8^{+}{ }_{(\mathrm{CT})}, \mathrm{CD}^{+}{ }_{(\mathrm{IM})}$, and $\mathrm{CD} 45 \mathrm{RO}^{+}{ }_{(\mathrm{IM})} \mathrm{TILs}$ was significantly lower than that for patients without recurrence (Fig. 4e-g). Surprisingly, the result for $\mathrm{CD} 8^{+}{ }_{(\mathrm{IM})}$ lymphocytes was similar to that for $\mathrm{CD}^{+}{ }_{(\mathrm{IM})}$ lymphocytes (Fig. $4 \mathrm{f}$ and i). Next, we verified the results for $\mathrm{CD}^{+}$and $\mathrm{CD}^{+} \mathrm{T}$ lymphocytes in the external validation cohort, wherein the patients achieved the same results as those of patients in the internal validation cohort (S14m-p and S17 Fig). Some unmentioned results are shown in Supplementary Fig. S14-17.

In summary, IRS reflects the infiltration of T lymphocytes into the IM of GC. This correlation may be attributed to the presence of $\mathrm{CD} 8^{+} \mathrm{T}$ lymphocytes. In the low- and middle-risk, an elevated IRS was accompanied by a decrease in $\mathrm{CD} 8^{+}{ }_{(\mathrm{IM})} \mathrm{T}$ lymphocytes, but this trend was the opposite in the high-risk.

\section{Increased IRS indicated suppression of the effect of CD8 ${ }^{+}$ (IM) T lymphocytes}

Abundant CD8 ${ }^{+}$lymphocytes were present in GC patients in the high-risk, but these patients did not have good prognoses. It has been reported that the co-positive of PD-1 and Tim-3 in T lymphocytes represent the hypo-functional one [28-30]. Therefore, we performed multiplexed immunofluorescence analysis for $45 \mathrm{GC}$ specimens to investigate the expression of PD-1 and Tim-3 in the three risk subgroups. Figure $5 \mathrm{a}$ shows the expression of PD-1 and TIM-3 in $\mathrm{CD}^{+}{ }_{(\mathrm{IM})}{ }^{\mathrm{T}}$ lymphocytes in the low-, middle, and high-risk. Approximately $28.8 \%$ and $18.8 \%$ of the $\mathrm{CD} 8^{+}$lymphocytes in the IM expressed PD- 1 and TIM-3, respectively, and $12.1 \%$ co-expressed those (Fig. $5 \mathrm{~b}$ and c). In the high-risk, PD- $1^{+} \mathrm{CD} 8^{+}{ }_{(\mathrm{IM})}$ and PD- $1^{+} \mathrm{TIM}-$ $3^{+} \mathrm{CD}^{+}{ }_{(\mathrm{IM})} \mathrm{T}$ lymphocytes displayed higher densities than those in the low- and middle-risk (Fig. $5 \mathrm{~d}$ ). The 
proportions of $\mathrm{CD}^{+}{ }_{(\mathrm{IM})} \mathrm{T}$ lymphocytes expressing these two inhibitory receptors in the three subgroups were also diverse. $\mathrm{CD}^{+}{ }_{(\mathrm{IM})}$ T lymphocytes showed the highest proportion of PD-1 or TIM-3 expression or a combination of the two in the high-risk (Fig. 5e). For the $\mathrm{CD}^{+}{ }_{(\mathrm{CT})} \mathrm{T}$ lymphocytes, there is no obvious difference (S18 Fig).

In summary, the IRS distinguished different characteristics of immune infiltration in the IM. Patients in the low-risk had the most abundant $\mathrm{CD} 8^{+}{ }_{(\mathrm{IM})} \mathrm{T}$ lymphocytes, a lower proportion of inhibitory receptor expression in $\mathrm{CD}^{+}$lymphocytes in the IM. For the middle-risk, the infiltration of $\mathrm{CD}^{+}{ }_{(\mathrm{IM})} \mathrm{T}$ lymphocytes was absent. Although the high-risk had increased infiltration of $\mathrm{CD}^{+}{ }_{(\mathrm{IM})} \mathrm{T}$ lymphocytes, the high proportion of PD-1 and TIM-3 expression caused most of these $\mathrm{CD} 8^{+}{ }_{(\mathrm{IM})} \mathrm{T}^{\mathrm{T}}$ lymphocytes to show an exhaustion pattern.

\section{Building a nomogram based on IRS to predict recurrence}

To facilitate the clinical application of IRS to predict recurrence in individual patients, we built a nomogram (Fig. 6a) based on the Cox regression model and integrated IRS and clinicopathological factors (T stage, $N$ stage, adjuvant chemotherapy, and the level of CA-199). In predicting 1-, 3- and 5-year recurrence, ROC analysis showed that the nomogram had high accuracy. The calibration analysis verified the reliability of the nomogram, and decision curve analysis (DCA) showed the clinical usefulness of the nomogram (Fig. 6b-d; S19 Fig).

Overall, for patients after GC resection, the IRS-based nomogram had better reliability, accuracy, and clinical availability in predicting recurrence. Furthermore, it was stable in predicting recurrence in different years. A nomogram based on IRS is more convenient for clinicians for formulating treatment and postoperative follow-up plans.

\section{Discussion}

The recurrence of GC is reportedly frequent but not uniform [42]. Up to $46.4 \%$ of GC patients relapse after curative resection in our center. Among the GC patients whose tumors re-emerged, the cumulative rates of recurrence within $1,2,3$, and 5 years were $38.8 \%, 69.4 \%, 80.1 \%$, and $92.1 \%$, respectively. Recently, immunotherapies such as anti-PD-1/PD-L1 have received considerable attention. Its combined use with conventional therapies has increased the survival rate of patients and highlighted the importance of immune factors surrounding the tumors $[17,18]$. Although PD-1/PD-L1 has become a potential immune marker for the prognosis and prediction of a series of malignant tumors, only $14.3-29.6 \%$ of GC patients express PD-L1, and the positive rate of PD-L1 in our center is less than 10\% [43-45]. Therefore, in addition to PD-L1, there are other immunosuppressive molecules that, alone or together with PD-L1, downregulate antitumor immunity. They promote immune suppression by reducing the infiltration of TILs, inhibiting $T$ cell activation, recruiting inhibitory cells such as Tregs or MDSCs, or increasing the expression of inhibitory receptors [35-38, 46-48]. 
The suppression of the immune microenvironment is considered to be one of the signs of GC progression and plays an important role in tumor re-appearance $[46,49]$. Therefore, indicators of immunosuppression may have predictive value for GC recurrence. However, different immunosuppressive indicators may be expressed in different tumors; it is therefore necessary to identify immunosuppressive indicators that play a role in GC. Based on our previous research, we identified seven immunosuppressive indicators that are significant in the prognosis of GC [41]. Lasso Cox regression analysis was used to fit recurrence data, eliminate the correlation between the indicators, and, finally, obtain the IRS based on six immunosuppressive indicators. The IRS reflects different risks of recurrence by classifying different immunosuppressive states.

Distinguishing the infiltration of TILs is critical for deconstructing the immunosuppressive microenvironment. Accumulating evidence indicates that TILs, which represent the local immune response [50-52], in many malignant tumors are related to the recurrence of several cancers [53-55]. Our research also confirmed that several immune biomarkers $\left(C D 3^{+}, C D 8^{+}, C D 45 R 0^{+}\right)$were significantly associated with recurrence (S14 Fig). However, this has a strong relationship with the location of TILs, and TILs at the IM seem to show more significant differences. Our data revealed that different IRS groups have different immune infiltration characteristics, and the level of IRS seems to be related to the effects of $\mathrm{CD} 8^{+}$TILs in the IM. Increased infiltration of $\mathrm{CD} 8^{+}$TILs enhances the local antitumor activity. In the low- and middle-risk groups, with the increase in IRS, the infiltration of CD8 ${ }^{+}$TILs in the IM tended to decrease. However, in the high-risk group, this trend was reversed. Although no reduction in the infiltration of $\mathrm{CD} 8^{+}$TILs was observed in the high-risk group, the $\mathrm{CD} 8^{+}$TILs in the high-risk group displayed noticeable exhaustion patterns, which seems to imply the loss of CD8 ${ }^{+}$TILs function. The lack of CD ${ }^{+}$ TILs in the IM may be linked to local recurrence [56]. We hypothesized that this may be due to the suppression of the local immune response, causing micrometastases to occur despite treatment. In our study, patients with local recurrence, especially in the perigastric lymph node group, had a higher IRS. This suggests that the IRS can effectively reflect the degree of local immune suppression. In summary, we termed the low-, middle-, and high-risk subgroups as immune-complete, immune-deficient, and immune-suppressive, respectively, which may contribute to selecting appropriate postoperative adjuvant treatment options.

Adjuvant chemotherapy is the standard adjuvant treatment for advanced resectable GC. Although chemotherapy can partially control the primary tumor, it provides little benefit for inhibiting the metastasis of cancer cells owing to the heterogeneity of tumors and the effect of suppressing immune surveillance $[57,58]$. A growing body of evidence has shown the efficacy of chemotherapy in addition to its direct cytostatic/cytotoxic effects but that it is also affected by the (re)activation of antitumor immune responses $[59,60]$. Chemotherapy can increase the immunogenicity of tumor cells and inhibit the immunosuppressive pathway of tumor formation [59]. It can also stimulate the anti-cancer immune response to kill residual tumor cells or residual micrometastases in the dormant stage [61]. In our study, IRS was able to predict the sensitivity of GC patients receiving adjuvant chemotherapy. In the middle-risk group, wherein the immune function was not completely suppressed, chemotherapy significantly reduced 
the recurrence rate, but in the high-risk group with immunosuppression, chemotherapy was ineffective. For these patients, new therapies need to be developed. Immunotherapy has advantages for a variety of cancers, and immunotherapy can improve the outcome of some patients who are resistant to chemotherapy [62-65]. At present, most patients who respond to immunotherapy have so-called "hot" tumors [66]. Hot tumors possess a high level of immune cell infiltration, but inhibitory receptors (such as PD-1, TIM-3) inhibit the antitumor effect $[20,22,66]$. Our data revealed that GC patients in the high-risk group were compatible with this feature; thus, we hypothesized that the prognoses of GC patients in the high-risk group might be improved through immunotherapy. Unfortunately, the current follow-up time of immunotherapy patients in our center is only 1 year, and there are no corresponding data to verify our hypothesis, which is the limitation of our study.

In this study, we defined a signature constructed using six immunosuppressive indicators to assess the risk of recurrence in GC patients. IRS can be treated as a classifier of local immune infiltration, which contributes to the selection of adjuvant treatment options for GC patients after resection. For patients in the low-risk group, the benefit of chemotherapy is not significant, and the decision to undergo chemotherapy should be determined according to individual conditions. Chemotherapy is recommended for patients in the middle-risk group. GC patients in the high-risk group who receive no benefit from chemotherapy alone may potentially benefit from immunotherapy. Our IRS and IRS-based nomogram showed significant prognostic value for recurrence and were verified in three independent cohorts. Our data also showed that GC patients with higher IRSs might be more likely to relapse in a short period of time after surgery and tend to relapse locoregionally, especially in the perigastric lymph nodes. Taken together, our results showed that the IRS can assist surgeons in the individualized selection of postoperative follow-ups, examinations, and treatment plans for patients. However, our research was based on retrospective data, and it is essential to verify it with prospective, multi-center clinical trials.

\section{Conclusion}

In general, we constructed an immunosuppressive status classifier based on immunosuppressive indicators that can be used to predict the recurrence and outcome of gastric cancer patients. Immunohistochemistry was used to obtain immunosuppressive recurrence score (IRS) to assess the risk of recurrence and patterns of recurrence after radical operation. Furthermore, the IRS has the potential as biomarker for selecting adjuvant treatment for GC patients.

\section{Abbreviations}

AJCC: American Joint Committee on Cancer; AUC: area under the curve; Cl: confidence interval; CT: center of tumor; DCA: decision curve analysis; DFS: diseases-free survival; EBV: Epstein-Barr virus; GC: gastric cancer; HR: hazard ratio; IM; invasive margin; IRS: immunosuppressive recurrence score; LASSO: least absolute shrinkage and selection operator; MDSCs: myeloid-derived suppressor cells; MSI: microsatellite instability; RFS: recurrence-free survival; PD-1: programmed cell death protein 1; PD-L1: programmed cell 
death 1 ligand 1; ROC: receiver operator characteristic; TAMs: tumor-associated macrophages; TILs: tumor-infiltrating lymphocytes; TIM-3: T cell immunoglobulin and mucin domain-containing protein 3;

\section{Declarations}

\section{Acknowledgments}

We thank the members of the Key Laboratory of the Ministry of Education for Gastrointestinal Cancer for their helpful comments and suggestions.

\section{Author contributions}

Jia-Bin Wang and Qing-Zhu Qiu conceived the study and drafted the manuscript. Jian-Wei Xie and ChangMing Huang helped critically revise the manuscript for important intellectual content. Qing-Zhu Qiu, NingZi Lian and Hua-Gen Wang performed the research. Qiao-Ling Zheng, Ying-Hong Yang, Yu-Bin Ma, Ya-Jun Zhao provide the samples. Ping Li, Jian-Xian Lin, Jun Lu, Qi-Yue Chen, Long-Long Cao and Mi Lin helped collect data and design the study.

\section{Availability of data and material}

The data supporting the findings in this study are available in the Article, Supplementary Information or from the corresponding author upon reasonable request.

\section{Competing interests}

The authors have declared no conflicts of interest.

\section{Consent for publication}

All patients obtained written informed consent before sample collection.

\section{Ethics approval and consent to participate}

The study was approved by the Ethics Committees of Fujian Medical University Union Hospital, Affiliated Hospital of Qinghai University, and First Affiliated Hospital of the University of Science and Technology of China. The ethics approval number of scientific research project is $2021 \mathrm{KY} 018$ from Fujian Medical University Union Hospital.

\section{Funding}

This work was supported by the National Natural Science Foundation of China (No. 81871899, 81802312, 82073187, 82073188), the Science and Technology Innovation Joint Fund Project of Fujian Province (No. 2019Y9089). 


\section{References}

1. Deng J, Liang H, Wang D, Sun D, Pan Y, Liu Y: Investigation of the recurrence patterns of gastric cancer following a curative resection. Surgery today 2011, 41(2):210-215.

2. Schwarz R, Zagala-Nevarez K: Recurrence patterns after radical gastrectomy for gastric cancer: prognostic factors and implications for postoperative adjuvant therapy. Annals of surgical oncology 2002, 9(4):394-400.

3. Yoo C, Noh S, Shin D, Choi S, Min J: Recurrence following curative resection for gastric carcinoma. The British journal of surgery 2000, 87(2):236-242.

4. D'Angelica M, Gonen M, Brennan MF, Turnbull AD, Bains M, Karpeh MS: Patterns of initial recurrence in completely resected gastric adenocarcinoma. Ann Surg 2004, 240(5):808-816.

5. Wu C, Lo S, Shen K, Hsieh M, Chen J, Chiang J, Lin H, Li A, Lui W: Incidence and factors associated with recurrence patterns after intended curative surgery for gastric cancer. World journal of surgery 2003, 27(2):153-158.

6. Sakuramoto S, Sasako M, Yamaguchi T, Kinoshita T, Fujii M, Nashimoto A, Furukawa H, Nakajima T, Ohashi Y, Imamura $\mathrm{H}$ et al: Adjuvant chemotherapy for gastric cancer with S-1, an oral fluoropyrimidine. The New England journal of medicine 2007, 357(18):1810-1820.

7. Smyth E, Verheij M, Allum W, Cunningham D, Cervantes A, Arnold D: Gastric cancer: ESMO Clinical Practice Guidelines for diagnosis, treatment and follow-up. Annals of oncology : official journal of the European Society for Medical Oncology 2016, 27:v38-v49.

8. Zitvogel L, Kepp O, Kroemer G: Immune parameters affecting the efficacy of chemotherapeutic regimens. Nature reviews Clinical oncology 2011, 8(3):151-160.

9. Casares N, Pequignot M, Tesniere A, Ghiringhelli F, Roux S, Chaput N, Schmitt E, Hamai A, HervasStubbs S, Obeid $\mathrm{M}$ et al: Caspase-dependent immunogenicity of doxorubicin-induced tumor cell death. The Journal of experimental medicine 2005, 202(12):1691-1701.

10. Apetoh L, Ghiringhelli F, Tesniere A, Criollo A, Ortiz C, Lidereau R, Mariette C, Chaput N, Mira J, Delaloge $S$ et al: The interaction between HMGB1 and TLR4 dictates the outcome of anticancer chemotherapy and radiotherapy. Immunological reviews 2007, 220:47-59.

11. Weber J, D'Angelo S, Minor D, Hodi F, Gutzmer R, Neyns B, Hoeller C, Khushalani N, Miller W, Lao C et al: Nivolumab versus chemotherapy in patients with advanced melanoma who progressed after antiCTLA-4 treatment (CheckMate 037): a randomised, controlled, open-label, phase 3 trial. The Lancet Oncology 2015, 16(4):375-384.

12. Kanda S, Goto K, Shiraishi H, Kubo E, Tanaka A, Utsumi H, Sunami K, Kitazono S, Mizugaki H, Horinouchi $\mathrm{H}$ et al: Safety and efficacy of nivolumab and standard chemotherapy drug combination in patients with advanced non-small-cell lung cancer: a four arms phase lb study. Annals of oncology : official journal of the European Society for Medical Oncology 2016, 27(12):2242-2250.

13. Nanda R, Liu M, Yau C, Shatsky R, Pusztai L, Wallace A, Chien A, Forero-Torres A, Ellis E, Han H et al: Effect of Pembrolizumab Plus Neoadjuvant Chemotherapy on Pathologic Complete Response in 
Women With Early-Stage Breast Cancer: An Analysis of the Ongoing Phase 2 Adaptively Randomized I-SPY2 Trial. JAMA oncology 2020, 6(5):676-684.

14. Burtness B, Harrington K, Greil R, Soulières D, Tahara M, de Castro G, Psyrri A, Basté N, Neupane P, Bratland $\AA$ et al: Pembrolizumab alone or with chemotherapy versus cetuximab with chemotherapy for recurrent or metastatic squamous cell carcinoma of the head and neck (KEYNOTE-048): a randomised, open-label, phase 3 study. Lancet (London, England) 2019, 394(10212):1915-1928.

15. Gutzmer R, Stroyakovskiy D, Gogas H, Robert C, Lewis K, Protsenko S, Pereira R, Eigentler T, Rutkowski P, Demidov $L$ et al: Atezolizumab, vemurafenib, and cobimetinib as first-line treatment for unresectable advanced BRAF mutation-positive melanoma (IMspire150): primary analysis of the randomised, double-blind, placebo-controlled, phase 3 trial. Lancet (London, England) 2020, 395(10240):1835-1844.

16. Robert C, Ribas A, Schachter J, Arance A, Grob J, Mortier L, Daud A, Carlino M, McNeil C, Lotem M et al: Pembrolizumab versus ipilimumab in advanced melanoma (KEYNOTE-006): post-hoc 5-year results from an open-label, multicentre, randomised, controlled, phase 3 study. The Lancet Oncology 2019, 20(9):1239-1251.

17. Pharmaceutical O: ONO submits supplemental application for approval for opdivo® (nivolumab) to expand the use for treatment of previously untreated unresectable advanced or recurrent gastric cancer in Japan. 2020, https://www.ono.co.jp/eng/news/pdf/sm_ cn200514.pdf, Accessed Aug 14, 2020.

18. Squibb B-M: Press release: CheckMate 649, a phase 3 trial evaluating opdivo (nivolumab) plus chemotherapy vs. chemotherapy, meets primary endpoints demonstrating superior overall survival and progressionfree survival in firstline treatment of gastric and esophageal cancers. 2020, https://news.bms.com/ pressrelease/corporatefinancialnews/checkmate649phase3trialevaluatingopdivonivolumabplusc, Accessed Aug 14, 2020.

19. Chen L, Han X: Anti-PD-1/PD-L1 therapy of human cancer: past, present, and future. The Journal of clinical investigation 2015, 125(9):3384-3391.

20. Chen YP, Zhang Y, Lv JW, Li YQ, Wang YQ, He QM, Yang XJ, Sun Y, Mao YP, Yun JP et al: Genomic Analysis of Tumor Microenvironment Immune Types across 14 Solid Cancer Types: Immunotherapeutic Implications. Theranostics 2017, 7(14):3585-3594.

21. Zeng D, Li M, Zhou R, Zhang J, Sun H, Shi M, Bin J, Liao Y, Rao J, Liao W: Tumor Microenvironment Characterization in Gastric Cancer Identifies Prognostic and Immunotherapeutically Relevant Gene Signatures. Cancer immunology research 2019, 7(5):737-750.

22. Tumeh P, Harview C, Yearley J, Shintaku I, Taylor E, Robert L, Chmielowski B, Spasic M, Henry G, Ciobanu $\mathrm{V}$ et al: PD-1 blockade induces responses by inhibiting adaptive immune resistance. Nature 2014, 515(7528):568-571.

23. Sun Y, Wu L, Zhong Y, Zhou K, Hou Y, Wang Z, Zhang Z, Xie J, Wang C, Chen D et al: Single-cell landscape of the ecosystem in early-relapse hepatocellular carcinoma. Cell 2021, 184(2):404421.e416. 
24. Jeong Y, Cho H, Kim T, Kim Y, Jeon S, Bychkov A, Jung C: CD73 Overexpression Promotes Progression and Recurrence of Papillary Thyroid Carcinoma. Cancers 2020, 12(10).

25. Hutchinson K, Yost S, Chang C, Johnson R, Carr A, McAdam P, Halligan D, Chang C, Schmolze D, Liang J et al: Comprehensive Profiling of Poor-Risk Paired Primary and Recurrent Triple-Negative Breast Cancers Reveals Immune Phenotype Shifts. Clinical cancer research : an official journal of the American Association for Cancer Research 2020, 26(3):657-668.

26. Corredor G, Wang X, Zhou Y, Lu C, Fu P, Syrigos K, Rimm DL, Yang M, Romero E, Schalper KA et al: Spatial Architecture and Arrangement of Tumor-Infiltrating Lymphocytes for Predicting Likelihood of Recurrence in Early-Stage Non-Small Cell Lung Cancer. Clin Cancer Res 2019, 25(5):1526-1534.

27. Wang M, Huang Y, Kong J, Sun Y, Tantalo D, Yeang H, Ying L, Yan F, Xu D, Halse H et al: Highdimensional analyses reveal a distinct role of T-cell subsets in the immune microenvironment of gastric cancer. Clinical \& translational immunology 2020, 9(5):e1127.

28. Henao-Tamayo M, Irwin S, Shang S, Ordway D, Orme I: T Iymphocyte surface expression of exhaustion markers as biomarkers of the efficacy of chemotherapy for tuberculosis. Tuberculosis (Edinburgh, Scotland) 2011, 91(4):308-313.

29. Liu J, Zhang S, Hu Y, Yang Z, Li J, Liu X, Deng L, Wang Y, Zhang X, Jiang T et al: Targeting PD-1 and Tim-3 Pathways to Reverse CD8 T-Cell Exhaustion and Enhance Ex Vivo T-Cell Responses to Autologous Dendritic/Tumor Vaccines. Journal of immunotherapy (Hagerstown, Md : 1997) 2016, 39(4):171-180.

30. Xu B, Yuan L, Gao Q, Yuan P, Zhao P, Yuan H, Fan H, Li T, Qin P, Han L et al: Circulating and tumorinfiltrating Tim-3 in patients with colorectal cancer. Oncotarget 2015, 6(24):20592-20603.

31. Kim K, Lee K, Cho H, Kim Y, Yang H, Kim W, Kang G: Prognostic implications of tumor-infiltrating FoxP3+ regulatory $\mathrm{T}$ cells and CD8+ cytotoxic T cells in microsatellite-unstable gastric cancers. Human pathology 2014, 45(2):285-293.

32. Chiaravalli A, Feltri M, Bertolini V, Bagnoli E, Furlan D, Cerutti R, Novario R, Capella C: Intratumour T cells, their activation status and survival in gastric carcinomas characterised for microsatellite instability and Epstein-Barr virus infection. Virchows Archiv : an international journal of pathology 2006, 448(3):344-353.

33. Liu K, Yang K, Wu B, Chen H, Chen X, Chen X, Jiang L, Ye F, He D, Lu Z et al: Tumor-Infiltrating Immune Cells Are Associated With Prognosis of Gastric Cancer. Medicine 2015, 94(39):e1631.

34. Li J, Byrne KT, Yan F, Yamazoe T, Chen Z, Baslan T, Richman LP, Lin JH, Sun YH, Rech AJ et al: Tumor Cell-Intrinsic Factors Underlie Heterogeneity of Immune Cell Infiltration and Response to Immunotherapy. Immunity 2018, 49(1):178-193 e177.

35. Huber V, Camisaschi C, Berzi A, Ferro S, Lugini L, Triulzi T, Tuccitto A, Tagliabue E, Castelli C, Rivoltini L: Cancer acidity: An ultimate frontier of tumor immune escape and a novel target of immunomodulation. Seminars in cancer biology 2017, 43:74-89.

36. He W, Zhang H, Han F, Chen X, Lin R, Wang W, Qiu H, Zhuang Z, Liao Q, Zhang W et al: CD155T/TIGIT Signaling Regulates CD8(+) T-cell Metabolism and Promotes Tumor Progression in Human Gastric 
Cancer. Cancer Res 2017, 77(22):6375-6388.

37. Kim W, Chu TH, Nienhuser H, Jiang Z, Del Portillo A, Remotti HE, White RA, Hayakawa Y, Tomita H, Fox JG et al: PD-1 Signaling Promotes Tumor-Infiltrating Myeloid-Derived Suppressor Cells and Gastric Tumorigenesis in Mice. Gastroenterology 2020.

38. Freeman G, Long A, Iwai Y, Bourque K, Chernova T, Nishimura H, Fitz L, Malenkovich N, Okazaki T, Byrne $\mathrm{M}$ et al: Engagement of the PD-1 immunoinhibitory receptor by a novel $\mathrm{B} 7$ family member leads to negative regulation of lymphocyte activation. The Journal of experimental medicine 2000 , 192(7):1027-1034.

39. Okoye I, Xu L, Motamedi M, Parashar P, Walker J, Elahi S: Galectin-9 expression defines exhausted T cells and impaired cytotoxic NK cells in patients with virus-associated solid tumors. Journal for immunotherapy of cancer 2020, 8(2).

40. Shen X, Zhao B: Efficacy of PD-1 or PD-L1 inhibitors and PD-L1 expression status in cancer: metaanalysis. BMJ (Clinical research ed) 2018, 362:k3529.

41. Wang JB, Li P, Liu XL, Zheng QL, Ma YB, Zhao YJ, Xie JW, Lin JX, Lu J, Chen QY et al: An immune checkpoint score system for prognostic evaluation and adjuvant chemotherapy selection in gastric cancer. Nat Commun 2020, 11(1):6352.

42. La Torre M, Rossi Del Monte S, Ferri M, Cosenza G, Mercantini P, Ziparo V: Peritoneal washing cytology in gastric cancer. How, when and who will get a benefit? A review. Minerva gastroenterologica e dietologica 2011, 57(1):43-51.

43. Dai C, Geng R, Wang C, Wong A, Qing M, Hu J, Sun Y, Lo A, Li J: Concordance of immune checkpoints within tumor immune contexture and their prognostic significance in gastric cancer. Molecular oncology 2016, 10(10):1551-1558.

44. Harada K, Dong X, Estrella J, Correa A, Xu Y, Hofstetter W, Sudo K, Onodera H, Suzuki K, Suzuki A et al: Tumor-associated macrophage infiltration is highly associated with PD-L1 expression in gastric adenocarcinoma. Gastric cancer : official journal of the International Gastric Cancer Association and the Japanese Gastric Cancer Association 2018, 21(1):31-40.

45. Tamura T, Ohira M, Tanaka H, Muguruma K, Toyokawa T, Kubo N, Sakurai K, Amano R, Kimura K, Shibutani $M$ et al: Programmed Death-1 Ligand-1 (PDL1) Expression Is Associated with the Prognosis of Patients with Stage II/III Gastric Cancer. Anticancer research 2015, 35(10):5369-5376.

46. Li X, Yao W, Yuan Y, Chen P, Li B, Li J, Chu R, Song H, Xie D, Jiang X et al: Targeting of tumourinfiltrating macrophages via CCL2/CCR2 signalling as a therapeutic strategy against hepatocellular carcinoma. Gut 2017, 66(1):157-167.

47. Thompson E, Zahurak M, Murphy A, Cornish T, Cuka N, Abdelfatah E, Yang S, Duncan M, Ahuja N, Taube $\mathrm{J}$ et al: Patterns of PD-L1 expression and CD8 T cell infiltration in gastric adenocarcinomas and associated immune stroma. Gut 2017, 66(5):794-801.

48. Liu Y, Liang X, Dong W, Fang Y, Lv J, Zhang T, Fiskesund R, Xie J, Liu J, Yin X et al: TumorRepopulating Cells Induce PD-1 Expression in CD8 T Cells by Transferring Kynurenine and AhR Activation. Cancer cell 2018, 33(3):480-494.e487. 
49. Zhou C, Weng J, Liu C, Zhou Q, Chen W, Hsu J, Sun J, Atyah M, Xu Y, Shi Y et al: High RPS3A expression correlates with low tumor immune cell infiltration and unfavorable prognosis in hepatocellular carcinoma patients. American journal of cancer research 2020, 10(9):2768-2784.

50. Cai T, Nesi G, Boddi V, Mazzoli S, Dal Canto M, Bartoletti R: Prognostic role of the tumor-associated tissue inflammatory reaction in transitional bladder cell carcinoma. Oncology reports 2006, 16(2):329-334.

51. Bremnes R, Al-Shibli K, Donnem T, Sirera R, Al-Saad S, Andersen S, Stenvold H, Camps C, Busund L: The role of tumor-infiltrating immune cells and chronic inflammation at the tumor site on cancer development, progression, and prognosis: emphasis on non-small cell lung cancer. Journal of thoracic oncology : official publication of the International Association for the Study of Lung Cancer 2011, 6(4):824-833.

52. Job S, Rapoud D, Dos Santos A, Gonzalez P, Desterke C, Pascal G, Elarouci N, Ayadi M, Adam R, Azoulay D et al: Identification of Four Immune Subtypes Characterized by Distinct Composition and Functions of Tumor Microenvironment in Intrahepatic Cholangiocarcinoma. Hepatology (Baltimore, Md) 2020, 72(3):965-981.

53. Quan H, Shan Z, Liu Z, Liu S, Yang L, Fang X, Li K, Wang B, Deng Z, Hu Y et al: The repertoire of tumor-infiltrating lymphocytes within the microenvironment of oral squamous cell carcinoma reveals immune dysfunction. Cancer immunology, immunotherapy : Cll 2020, 69(3):465-476.

54. Chen F, Yang Y, Zhao Y, Pei L, Yan H: Immune Infiltration Profiling in Nonsmall Cell Lung Cancer and Their Clinical Significance: Study Based on Gene Expression Measurements. DNA and cell biology 2019, 38(11):1387-1401.

55. Takami H, Fukushima S, Aoki K, Satomi K, Narumi K, Hama N, Matsushita Y, Fukuoka K, Yamasaki K, Nakamura $\mathrm{T}$ et al: Intratumoural immune cell landscape in germinoma reveals multipotent lineages and exhibits prognostic significance. Neuropathology and applied neurobiology 2020, 46(2):111-124.

56. Zlobec I, Terracciano LM, Lugli A: Local recurrence in mismatch repair-proficient colon cancer predicted by an infiltrative tumor border and lack of CD8+ tumor-infiltrating lymphocytes. Clin Cancer Res 2008, 14(12):3792-3797.

57. Fernald K, Kurokawa M: Evading apoptosis in cancer. Trends in cell biology 2013, 23(12):620-633.

58. Zitvogel L, Tesniere A, Kroemer G: Cancer despite immunosurveillance: immunoselection and immunosubversion. Nature reviews Immunology 2006, 6(10):715-727.

59. Galluzzi L, Buqué A, Kepp O, Zitvogel L, Kroemer G: Immunological Effects of Conventional Chemotherapy and Targeted Anticancer Agents. Cancer cel/2015, 28(6):690-714.

60. Coffelt S, de Visser K: Immune-mediated mechanisms influencing the efficacy of anticancer therapies. Trends in immunology 2015, 36(4):198-216.

61. Zitvogel L, Apetoh L, Ghiringhelli F, André F, Tesniere A, Kroemer G: The anticancer immune response: indispensable for therapeutic success? The Journal of clinical investigation 2008, 118(6):1991-2001.

62. Powles T, Plimack E, Soulières D, Waddell T, Stus V, Gafanov R, Nosov D, Pouliot F, Melichar B, Vynnychenko I et al: Pembrolizumab plus axitinib versus sunitinib monotherapy as first-line 
treatment of advanced renal cell carcinoma (KEYNOTE-426): extended follow-up from a randomised, open-label, phase 3 trial. The Lancet Oncology 2020, 21(12):1563-1573.

63. André T, Shiu K, Kim T, Jensen B, Jensen L, Punt C, Smith D, Garcia-Carbonero R, Benavides M, Gibbs $\mathrm{P}$ et al: Pembrolizumab in Microsatellite-Instability-High Advanced Colorectal Cancer. The New England journal of medicine 2020, 383(23):2207-2218.

64. Brastianos P, Lee E, Cohen J, Tolaney S, Lin N, Wang N, Chukwueke U, White M, Nayyar N, Kim A et al: Single-arm, open-label phase 2 trial of pembrolizumab in patients with leptomeningeal carcinomatosis. Nature medicine 2020, 26(8):1280-1284.

65. Gao J, Navai N, Alhalabi O, Siefker-Radtke A, Campbell M, Tidwell R, Guo C, Kamat A, Matin S, Araujo $\mathrm{J}$ et al: Neoadjuvant PD-L1 plus CTLA-4 blockade in patients with cisplatin-ineligible operable highrisk urothelial carcinoma. Nature medicine 2020, 26(12):1845-1851.

66. Galon J, Bruni D: Approaches to treat immune hot, altered and cold tumours with combination immunotherapies. Nature reviews Drug discovery 2019, 18(3):197-218.

\section{Figures}
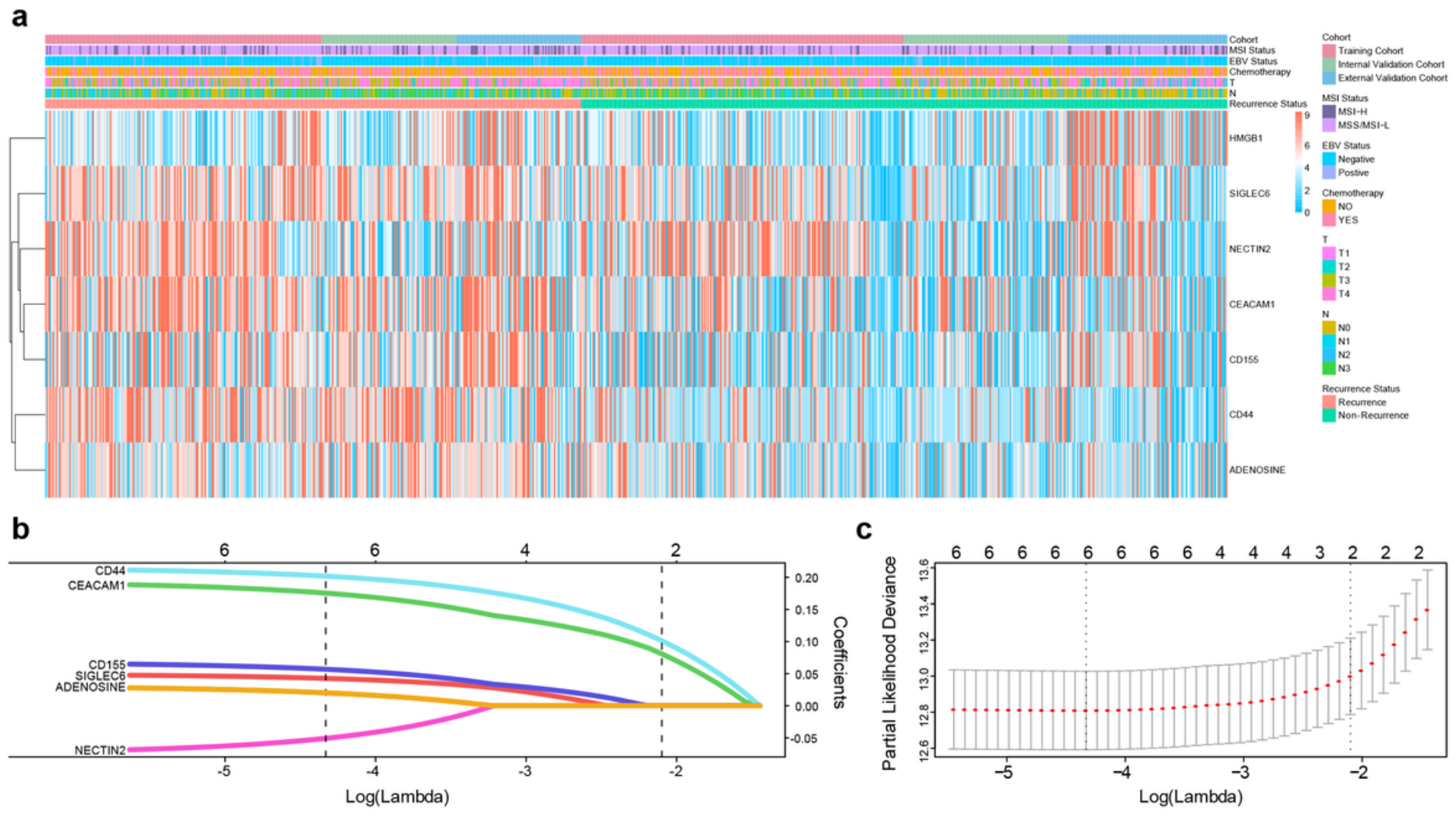

b
C

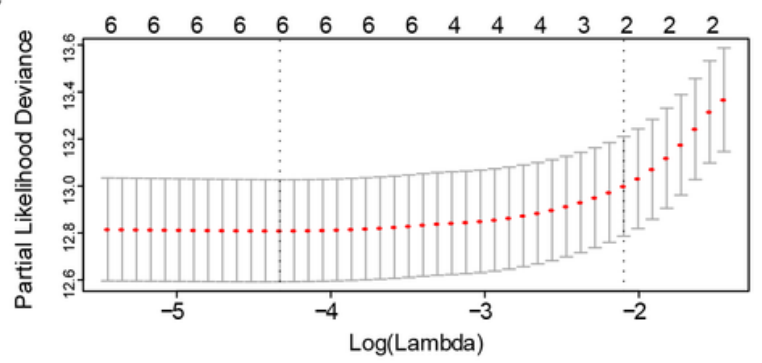

Figure 1

Construction of the immunosuppressive recurrence score (IRS). a, Heatmap showing immunohistochemistry scores of seven immunosuppressive checkpoints (HMGB1, SIGLEC6, NECTIN2, CEACAM1, CD155, CD44, and ADENOSINE) in the training cohort $(n=418)$, internal validation cohort $(n=$ 
209), and external validation cohort $(n=198)$. b, Construction of the IRS by the lasso Cox regression method; the Lasso coefficient of six immunosuppressive checkpoints is shown. c, Ten-fold crossvalidation of fine-tuning parameter selection in the lasso model.

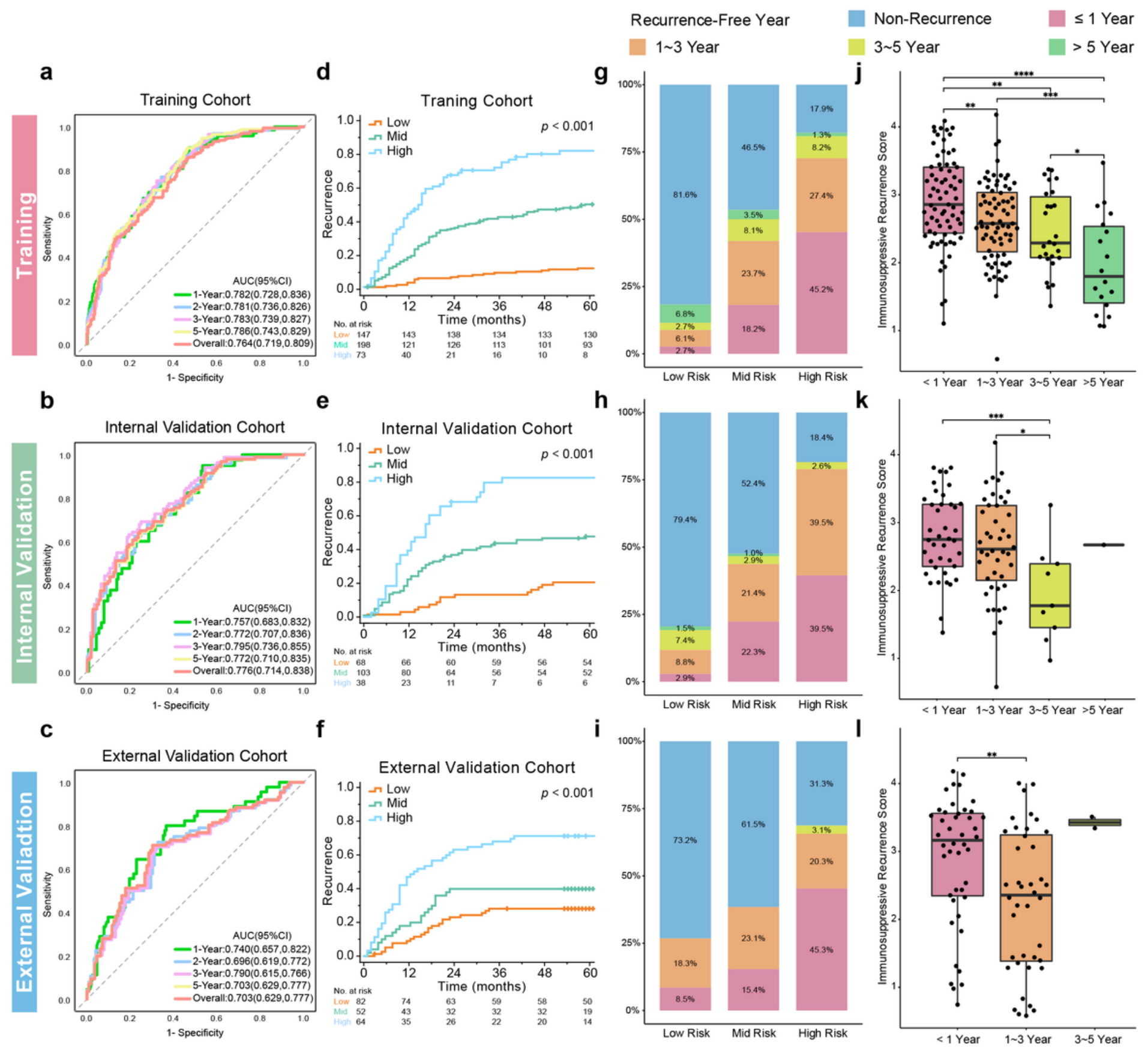

Figure 2

Validation of immunosuppressive recurrence score (IRS) in the training $(n=418)$, internal validation $(n=$ 209), and external validation ( $n=198)$ cohorts. a-c, Time-dependent receiver operator characteristic (ROC) curves of IRS for recurrence-free survival (RFS) in the training, internal validation and external validation cohorts. b, Kaplan-Meier curves for RFS according to IRS in the training, internal validation and external validation cohorts. c, Compositions of recurrence status and recurrence time in the low-risk, middle-risk, and high-risk in the training, internal validation and external validation cohorts. $P<0.001, P<0.001, P=$ 
0.007, respectively (Fisher's exact test). d, Comparison of IRS between gastric cancer (GC) patients with different recurrence times in the training, internal validation and external validation cohorts. ${ }^{\star} P<0.05$; $* * P$ $<0.01 ; * \star * P<0.001 ; * \star * * \mathrm{P}<0.0001$; non-significant values are shown as blanks (Kruskal-Wallis test).

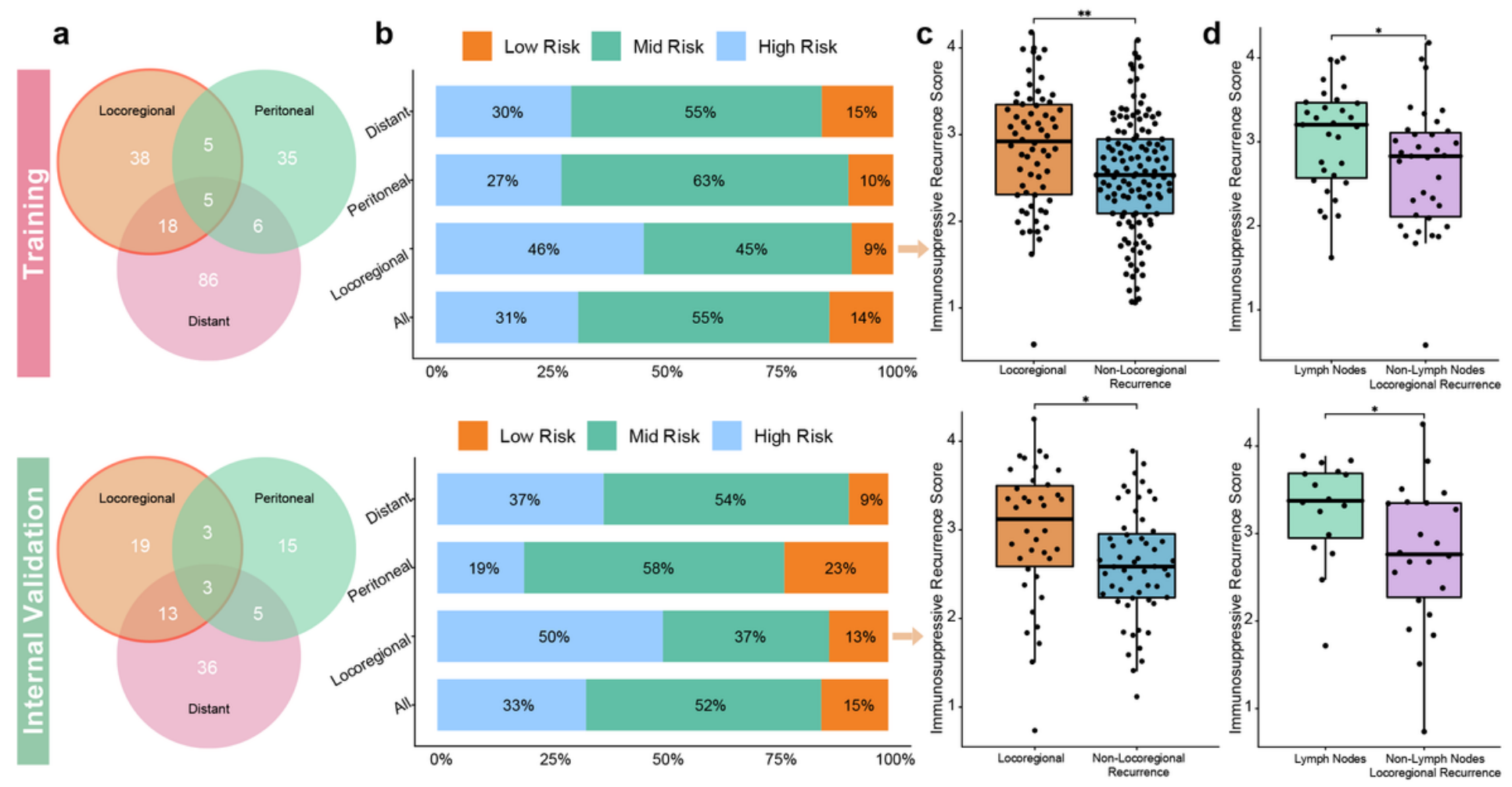

Figure 3

Association of immunosuppressive recurrence score (IRS) with different patterns of recurrence. a, Venn diagram of recurrence patterns in the internal cohorts with documented recurrence. b, Proportions of three subgroups (low-risk, middle-risk, and high-risk subgroups) of patients with different recurrence patterns in the internal cohorts. c, Comparison of IRS between patients with locoregional recurrence and patients with non-locoregional recurrence in the internal cohorts. d, Among all gastric cancer (GC) patients with locoregional recurrence, comparison of IRS between patients with recurrence in lymph node and patients with non-lymph node locoregional recurrence in the internal cohorts. ${ }^{*} \mathrm{P}<0.05$; ${ }^{\star \star} \mathrm{P}<0.01$ (Mann-Whitney test). 

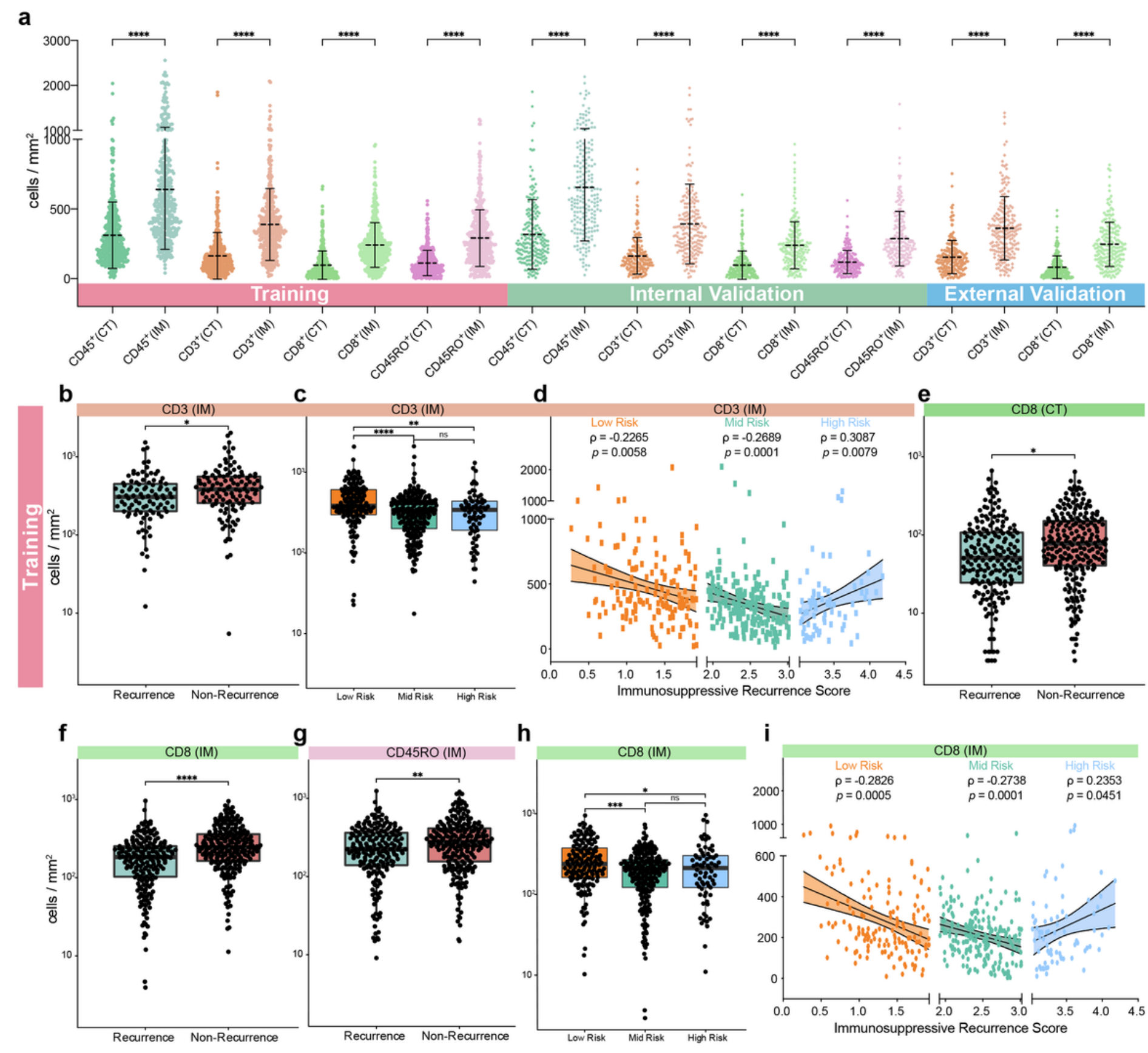

\section{Figure 4}

Association of immunosuppressive recurrence score (IRS) with infiltration of immune biomarkers. a, Expression of four immune biomarkers (CD45+, CD3+, CD8+, CD45RO+) in the center of the tumor (CT) and invasive margin (IM) of gastric cancer (GC) patients from the internal cohorts $(n=627)$, and the expression of two immune biomarkers (CD3+, CD8+) in the CT and IM of GC patients from the external validation cohort $(n=198)$. ${ }^{\star \star * *} \mathrm{P}<0.0001$ (paired $t$ test). Error bar indicates mean and standard deviation. $b$, Comparison of the expression of $\mathrm{CD} 3+(\mathrm{IM})$ in $\mathrm{GC}$ patients with and without recurrence from the training cohort. ${ }^{*} \mathrm{P}<0.05$ (Mann-Whitney test). $\mathrm{C}$, Comparison of the expression of $\mathrm{CD} 3+(\mathrm{IM})$ in three subgroups (low-risk, middle-risk, and high-risk subgroups) of GC patients from the training cohort. ${ }^{\star \star} \mathrm{P}<0.01$; $* \star \star \star \mathrm{P}<$ 0.0001 (Kruskal-Wallis test). $d$, Correlation between IRS values and infiltration levels of CD3+(IM) in the 
training cohort of GC patients (Spearman correlation test). e-g, Comparison of the expression of CD8+ (CT), CD8+(IM), and CD45RO+(IM) in GC patients with and without recurrence from the training cohort. *P $<0.05$; ${ }^{\star *} \mathrm{P}<0.01$; $* \star \star \star P<0.0001$ (Mann-Whitney test). $h$, Comparison of the expression of CD8+(IM) in three subgroups (low-risk, middle-risk, and high-risk subgroups) of GC patients from the training cohort (n $=418) .{ }^{*} \mathrm{P}<0.05 ;{ }^{*} * \mathrm{P}<0.001$ (Kruskal-Wallis test). $\mathrm{i}$, Correlation between IRS values and infiltration levels of CD8+(IM) in the training cohort of GC patients (Spearman correlation test).

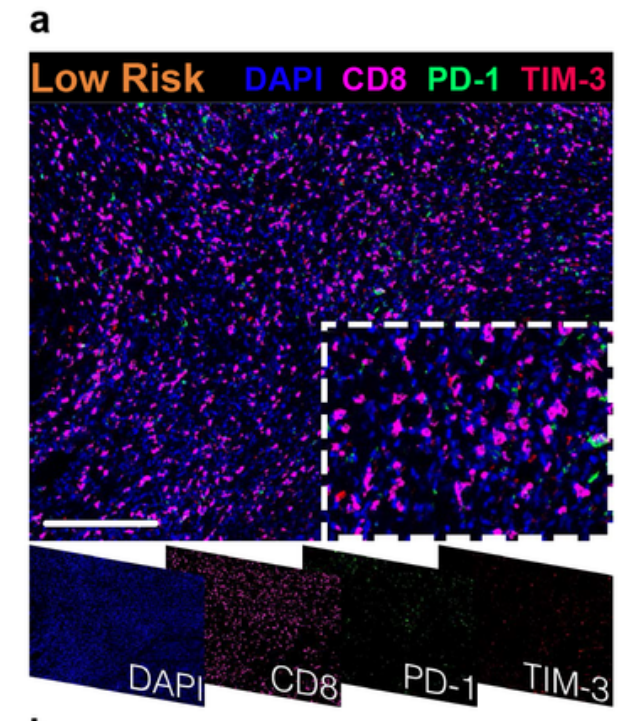

b
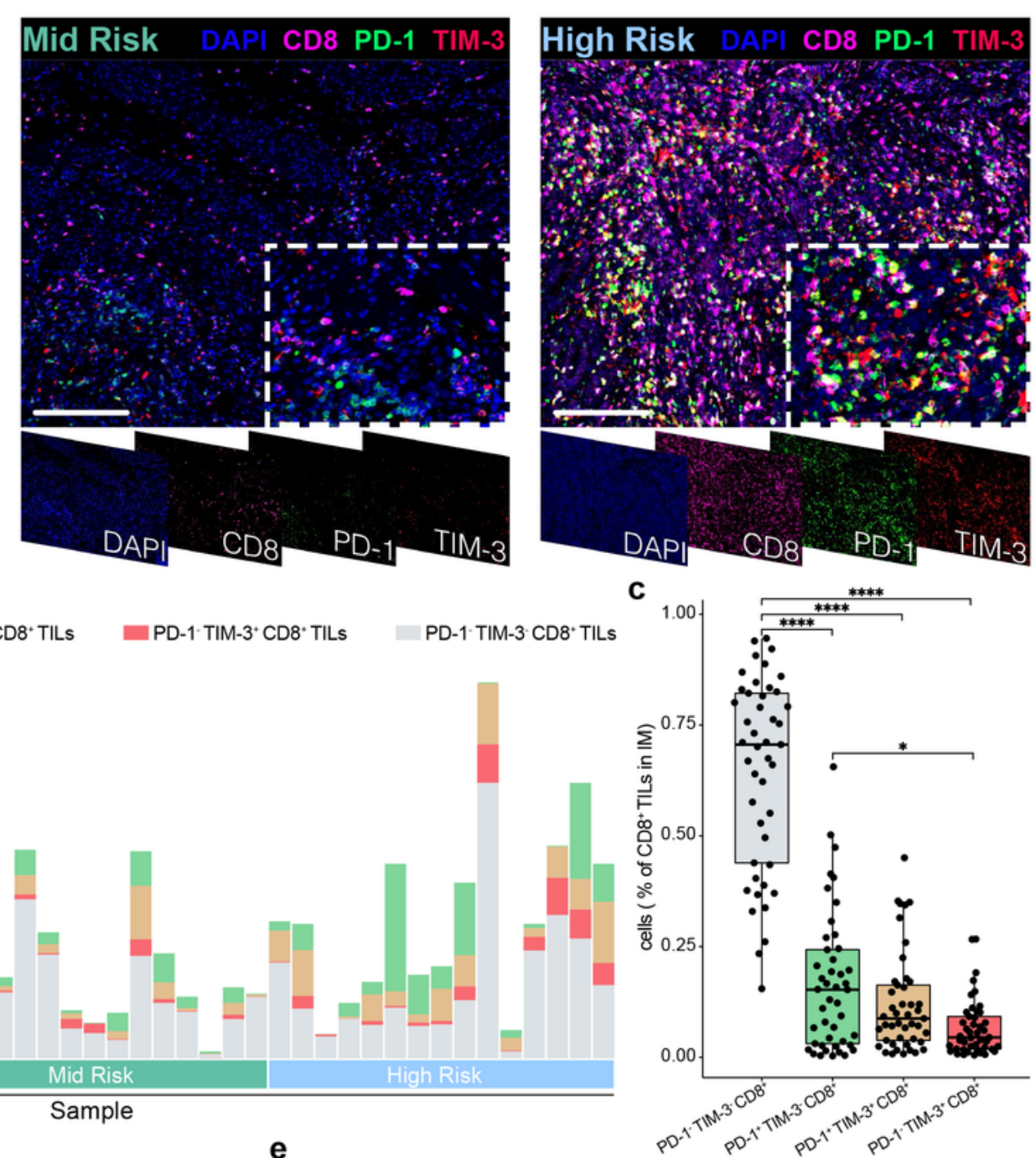

d
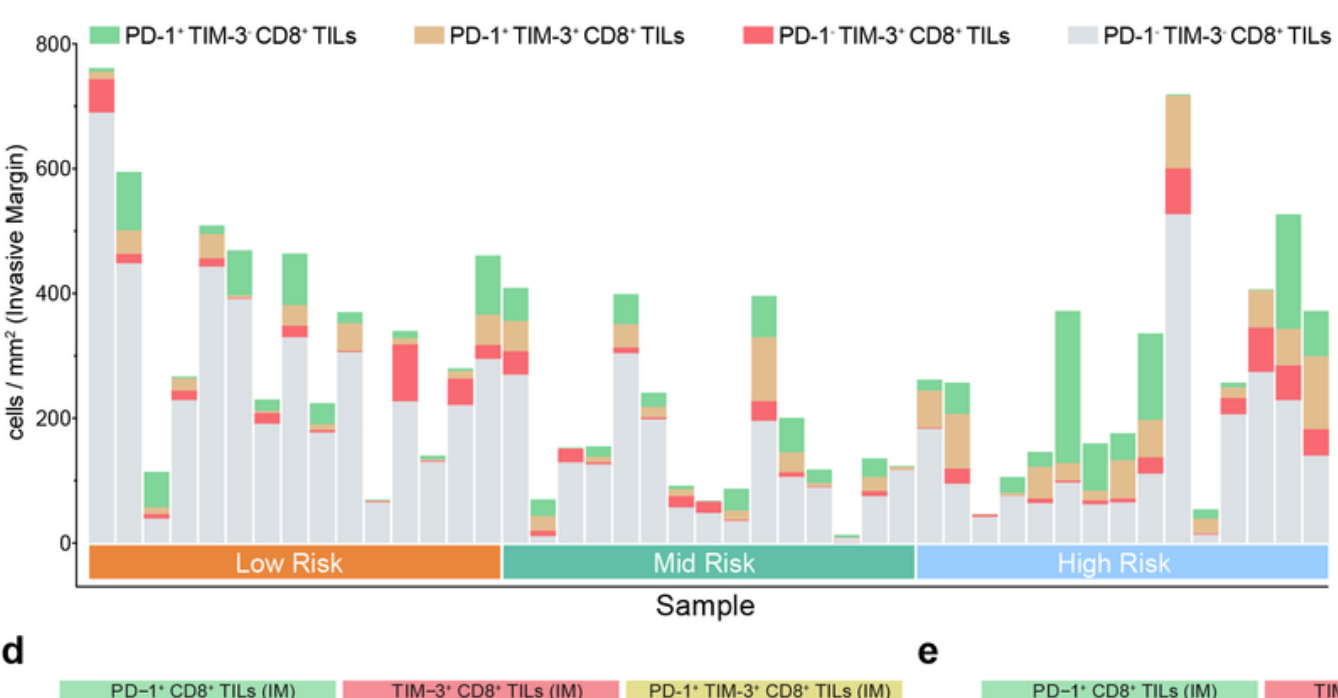

PD-1. CD8. TILS (IM) TIM-3. CD8. TILS (IM) PD-1* TIM-3. CD8* TILS (IM)
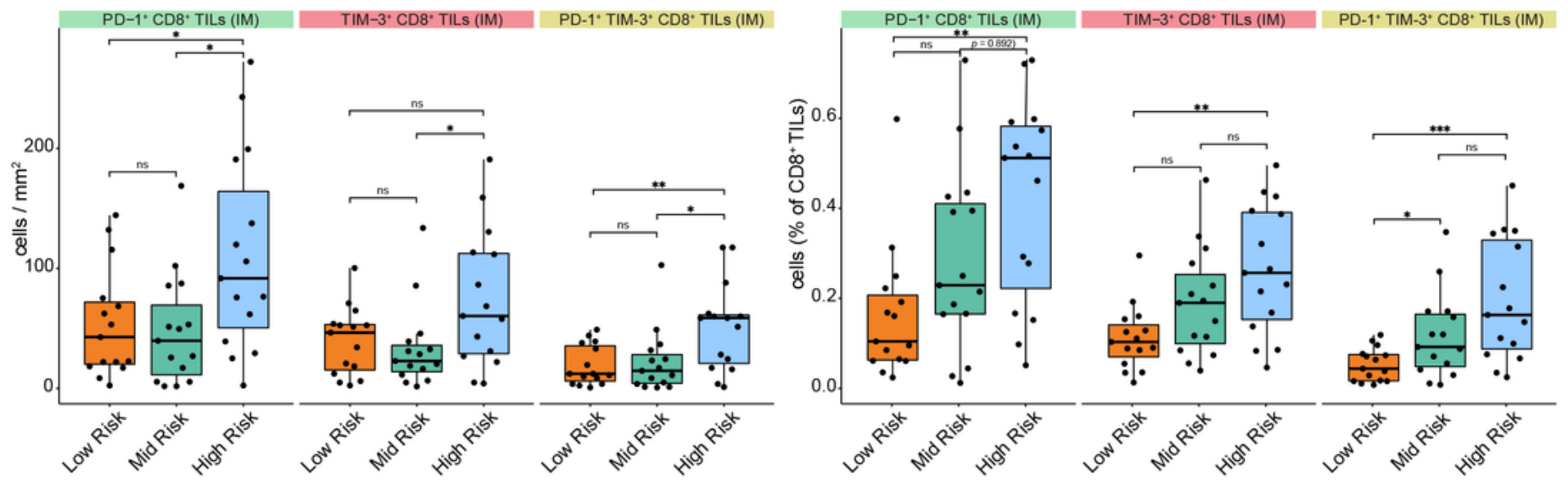

Figure 5 
Multiplexed immunofluorescence (IF) staining revealed the expression of immune checkpoints (PD-1 and TIM-3) in the invasive margin (IM) in the three risk subgroups. a, Representative multiplexed IF images showing the expression of PD-1 and TIM-3 in CD8+ lymphocytes in the three risk subgroups of gastric cancer (GC) patients. b, In the low-risk $(n=15)$, middle-risk $(n=15)$, and high-risk $(n=15)$ groups, the different subtypes of CD8+ lymphocytes were classified according to the expression of PD-1 and TIM-3. C, Percentages of PD-1-TIM-3-CD8+(IM), PD-1+TIM-3-CD8+(IM), PD-1+TIM-3+CD8+(IM) and PD-1-TIM+CD8+ (IM) T lymphocytes in $45 \mathrm{GC}$ patients. $d$, Comparison of the expression of PD-1+CD8+(IM), TIM-3+CD8+ $(I M)$, and PD-1+TIM-3+CD8+(IM) in the low-risk $(n=15)$, middle-risk $(n=15)$, and high-risk $(n=15)$ risk groups of GC patients. e, Comparison of the percentages of CD8+ lymphocytes expressing PD-1+CD8+ (IM), TIM-3+CD8+(IM), and PD-1+TIM-3+CD8+(IM) in the low-risk $(n=15)$, middle-risk $(n=15)$, and high-

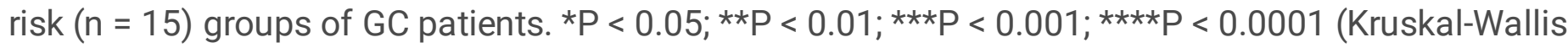
test). Scale bar $=200 \mu \mathrm{m}$.

a

Points

IRS

Depth of Invasion

Lymph Node Metastasis

Adjuvant Chemotherapy

CA19-9

Total Points

1-Year Recurrence Probability

3-Year Recurrence Probability

5-Year Recurrence Probability

d
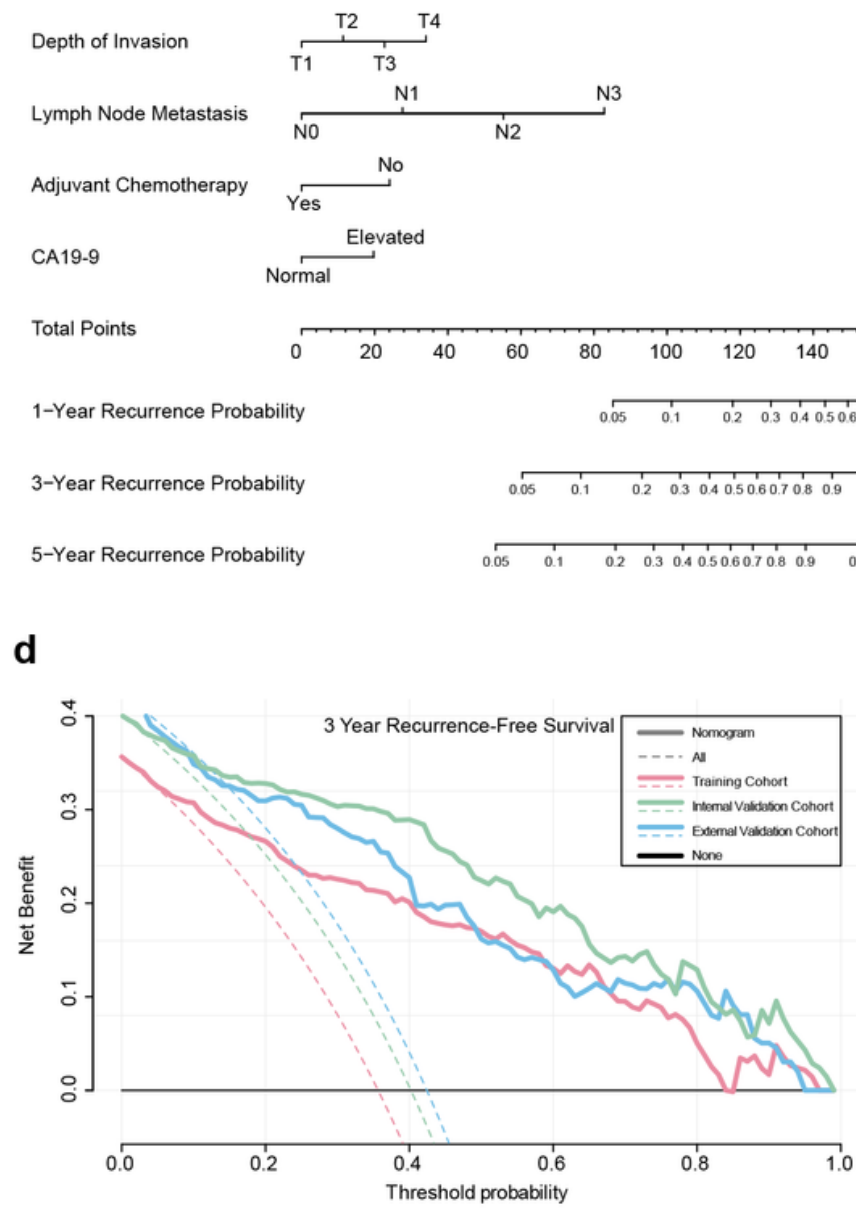

d b
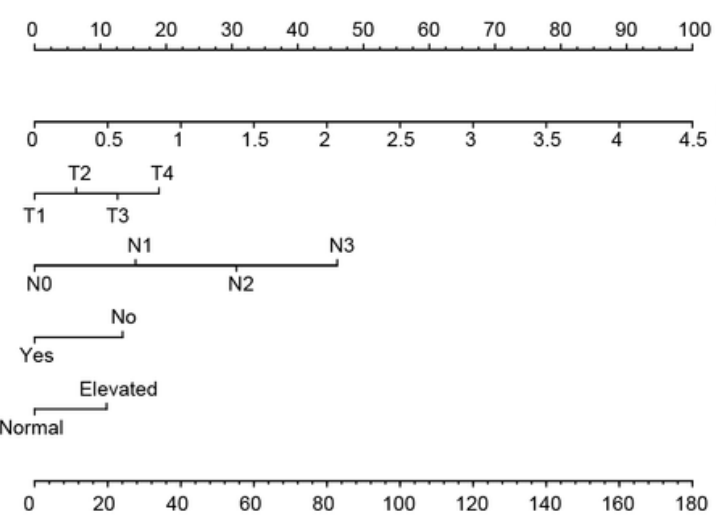

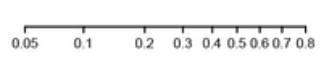
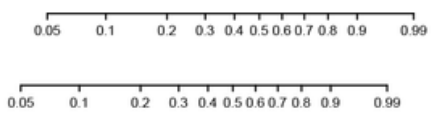

\section{Figure 6}

Construction of a nomogram based on the immunosuppressive recurrence score (IRS) to predict recurrence probability. a, Nomogram based on the IRS to predict postoperative recurrence. b-d, Time- 
dependent ROC curve, calibration curve and DCA for the nomogram in the training cohort and in the internal, and external validation cohorts.

\section{Supplementary Files}

This is a list of supplementary files associated with this preprint. Click to download.

- SupplementaryFigureS1S19andTableS1S7.pdf

- SupplementaryMethods.pdf 